\title{
QuTE: Answering Quantity Queries from Web Tables
}

\author{
Vinh Thinh Ho \\ hvthinh@mpi-inf.mpg.de \\ Max Planck Institut für Informatik \\ Saarbrücken, Germany
}

\author{
Koninika Pal \\ kpal@mpi-inf.mpg.de \\ Max Planck Institut für Informatik \\ Saarbrücken, Germany
}

\author{
Gerhard Weikum \\ weikum@mpi-inf.mpg.de \\ Max Planck Institut für Informatik \\ Saarbrücken, Germany
}

\begin{abstract}
Quantities are financial, technological, physical and other measures that denote relevant properties of entities, such as revenue of companies, energy efficiency of cars or distance and brightness of stars and galaxies. Queries with filter conditions on quantities are an important building block for downstream analytics, and pose challenges when the content of interest is spread across a huge number of web tables and other ad-hoc datasets. Search engines support quantity lookups, but largely fail on quantity filters. The QuTE system presented in this paper aims to overcome these problems. It comprises methods for automatically extracting entity-quantity facts from web tables, as well as methods for online query processing, with new techniques for query matching and answer ranking.

\section{ACM Reference Format:}

Vinh Thinh Ho, Koninika Pal, and Gerhard Weikum. 2021. QuTE: Answering Quantity Queries from Web Tables. In Proceedings of the 2021 International Conference on Management of Data (SIGMOD '21), fune 18-27, 2021, Virtual Event, China. ACM, New York, NY, USA, 5 pages. https://doi.org/10.1145/ 3448016.3452763
\end{abstract}

\section{INTRODUCTION}

Motivation. Searching for entities with filter conditions on quantitative properties, like financial, physical or technological measures, is common in database applications and well supported. For Internet contents, including text and tables in web pages, however, search engines often fail to provide answers as they do not understand quantity values and their units and do not infer which quantity refers to which entity. The following are example queries that an analyst or data scientist could pose:

- British football teams worth more than 1 Billion Euros

- cities with annual energy consumption above $50 \mathrm{TWh}$

- European football stadiums with more than 60000 seats

For such queries, search engines sometimes return pages with largest/smallest/richest/etc. entities, such as Wikipedia lists, but this depends on the keywords and the quantity value in the query. For example, "celebrities worth more than 1 billion" returns a list, but the seemingly minor variations "... above 1.5 billion" or "women worth ..." fall back to merely giving ten blue links to all kinds of web pages - some off topic, some worthwhile reading but still needing human effort to identify answer entities within long documents. Note that these kinds of queries with quantity filters are different

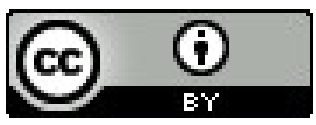

This work is licensed under a Creative Commons Attribution International 4.0 License. SIGMOD '21, Fune 18-27, 2021, Virtual Event, China.

(C) 2021 Copyright held by the owner/author(s).

ACM ISBN 978-1-4503-8343-1/21/06.

https://doi.org/10.1145/3448016.3452763 from quantity lookups, such as "net worth of Rihanna". Lookups are much simpler and handled fairly well by search engines and QA assistants; they do not involve the interpretation of filter conditions.

Structured data and knowledge bases, accessible through web APIs, could be considered as a solution. However, the relevant databases are scattered across the Internet, posing obstacles in discovering them and understanding their schemas, options for joins and unions, trustworthiness, etc. Knowledge bases, such as Wikidata, have good coverage of entities, but barely contain quantitative properties (e.g., 2000 marathon runners but only 20 with their best time captured, 3000 car models but none with engine power, energy efficiency, carbon footprint, etc.).

Approach. The research that led to this system demo proposal pursues the goal of supporting web-based quantity queries, bringing database functionality to unstructured and semi-structured content. Our work includes tapping into textual contents $[5,6]$ as well as web tables (embedded in HTML pages) [7]. This demo paper is about the latter.

We present the QuTE system, which taps into web tables to answer quantity queries. It has two major components: 1) extracting entity-quantity facts from tables using machine-learning techniques, and 2) matching queries against an indexed repository of such facts and computing ranked answer lists. The extraction component is described in detail in [7]. The query processor is based on techniques originally developed in [5] for the different case of quantitites in text documents. For the case of web tables, we devised several extensions, in particular, for contextualizing quantity facts and for enriching them with evidence from text corpora to enhance the answer ranking. This demo largely focuses on the query processing part. The QuTE system can be accessed at https://qsearch.mpi-inf.mpg.de/table/.

\section{SYSTEM OVERVIEW}

We refer to the entity-quantity facts that QuTE taps into as Qfacts: triples of the form (entity, quantity, context) where the context consists of words and phrases that add evidence and explanation to the fact (e.g., (Anfield, 61905, "record attendance 1952")). The two main stages of QuTE are the extraction of Qfacts from web tables, carried out as an offline computation, and the online query processing. The first stage comprises components for entity linking, quantity normalization, column alignment, and Qfact contextualization. The second stage involves matching and ranking of Qfacts against user queries. Figure 1 gives a pictorial overview.

Entity Linking. The processing of web tables starts with detecting entities and quantities. These are standard tasks for text analysis. Entities are disambiguated by linking them to the Yago knowledge graph, by inference based on a probabilistic factor graph (similar to $[2])$. 
Table 1: Example table: entities and quantities are in light green and red, respectively; arrows indicate the column alignment.

\begin{tabular}{|c|c|c|c|c|c|c|c|c|c|c|}
\hline & & & & & & & $\sqrt{2}$ & & & \\
\hline Team & Stadium & Capacity & Coach & Value (in Bio) & & Team & Stadium & Capacity & Coach & Value (in Bio) \\
\hline Bayern & Allianz Arena & ca. 75000 & Hansi Flick & 2.549 Euro & & $<$ FC_Bayern_Munich> & $<$ Allianz_Arena $>$ & ca. $(75000 ;)$ & $<$ Hansi_Flick $>$ & $(2.549 \mathrm{e} 9 ; €)$ \\
\hline & Bernabéu & 81,044 & Zidane & 3.649 Euro & $\Longrightarrow$ & $<$ FC_Real_Madrid > & $<$ Santiago_Bernabéu $>$ & $(81044 ;)$ & <Zinedine_Zidane> & $(3.649 \mathrm{e} 9 ; €)$ \\
\hline Man City & unknown & $\mathrm{n} / \mathrm{a}$ & Pep Guardiola & $2.055 \mathrm{GBP}$ & & <FC_Manchester_City > & unknown & & <Pep_Guardiola> & $(2.055 \mathrm{e} 9 ; £)$ \\
\hline Chelsea & Stamford Bridge & 40,834 & Frank Lampard & $1.958 \mathrm{GBP}$ & & $<$ FC_Chelsea $>$ & $<$ Stamford_Bridge $>$ & (40834;) & $<$ Frank_Lampard $>$ & $(1.958 \mathrm{e} 9 ; \mathfrak{f})$ \\
\hline
\end{tabular}

Quantity Normalization. This step involves detecting numerical expressions and inferring their units when applicable, e.g., from column headers. We build on rule-based prior works $[12,13]$, with some extensions. We can now easily classify table columns into Ecolumns (mostly rows with entities), Q-columns (mostly quantities) and Others (e.g., text comments).

Column Alignment. For meaningful Qfacts, we need to go beyond the above extraction steps, and compute which quantity denotes a property of which entity. This is challenging for complex tables with more than one E-column. Table 1 shows an example. Upfront, it is unclear whether Capacity refers to Team or Stadium and whether Value is a property of Team, Stadium or Coach. In [7] we have devised a novel solution for this column alignment problem, with much higher precision than prior baselines $[3,11,18]$. In a nutshell, we compute co-occurrence scores for entity-quantity pairs for candidate alignments, aggregating over the rows of the two columns. The co-occurrences are obtained from proximitybased matching in a large text corpus (Wikipedia articles and other web content). Details are in [7].

Contextualization. Bare Qfacts are not sufficient for effective search, as user queries often contain cues about the measure of interest and all kinds of modifiers. For example, the query "British football teams worth more than 1 Billion Euros" includes the measure "worth" and the modifier "British". Therefore, we augment each of the extracted entity-quantity pairs with context words derived from the column headers, the table caption, the other values in the same row, the page title, the labels on the DOM-tree path to the table (e.g., intermediate headings) and table surrounding text.

Query Matching. User queries are decomposed into the answer type (e.g., football teams), the quantity condition (e.g., $\geq 1,000,000,000$ Euro) and contextual cues. These three components are matched against the (millions of) Qfacts from the extraction stage. For the context word matching, we use IR-style language models with word embeddings, so that semantically related matches are captured as well (e.g., "worth" matching "value"). Section 3 gives more detail.

Answer Ranking. The ranking of the returned answers is mostly based on the text-matching scores for the query and Qfact contexts. In addition, we devised two novel components that provide signals for corroboration and enhanced ranking. First, we identify witnesses for the confidence in a Qfact answer, in the form of evidence snippets from a large corpus of Wikipedia articles and web pages. These are automatically retrieved by contextualized search, at query runtime. Second, we compute a score for the mutual consistency of candidate answers, reflecting their agreement on quantity values in the same order of magnitude and on related context words. These two kinds of signals are added to the context-matching scores with hyper-parameter weights.

\section{QUERY MATCHING}

Queries are matched against the repository of Qfacts by 1) testing that entities belong to the semantic type stated in the query (using the rich type system of the Yago knowledge base), 2) evaluating the quantity filter condition, and 3) matching all cue words in the query against the precomputed context of the candidate Qfacts. The Qfact context is obtained from six regions of the web page:

1. headers of the E-column and Q-column where the fact appeared

2. the page title,

3. labels in the DOM-tree path to the table,

4. the table caption,

5. all other cells of the same table row,

6. text surrounding the table, within a window of 100 words.

The QuTE demo highlights all of these contextual cues. Extensive experiments, with tuning of hyper-parameter weights, showed that the regions 1 and 2 are by far the most valuable [7]. For the regions 3 to 6 , the noise-to-signal ratio is often high.

For the degree of matching query cues $Q$ against Qfact context $X$, QuTE employs a language-model-based scoring function. This incorporates word2vec embeddings to capture the influence of semantically related words (e.g., "worth" and "value" being near-matches). Specifically, we use the following weighted directed embedding distance (wded), originally proposed in [5] and adapted to the new setting of web tables:

$$
\operatorname{wded}(Q, X)=\left(\sum_{u \in Q} \omega(u) \cdot \min _{v \in X}(d(u, v) \cdot L(v))\right) /\left(\sum_{u \in Q} \omega(u)\right)
$$

where $\omega$ are tf-idf weights of tokens, $d(u, v)$ is the cosine between word embeddings, and $L(v)$ denotes the weight of token $v$ depending on which region it is collected from.

\section{ANSWER RANKING}

The ranking of candidate answers is primarily computed by the matching score (see Section 3). In addition, we harness two unconventional signals to further enhance the ranking.

Evidence from Text Corpus. As the context of table-based Qfacts is often sparse or noisy, QuTE has a new technique of collecting

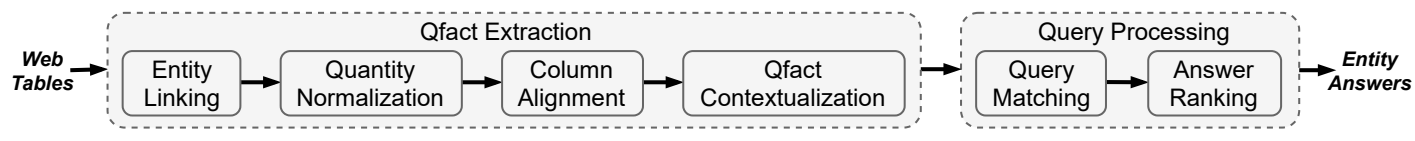

Figure 1: System architecture of QuTE. 
witnesses for Qfact validity by retrieving evidence from a large text corpus of Wikipedia articles and other pages from a large news corpus, a total of 13.5 Million documents.

This evidence collection is performed for each answer candidate $A$ at query time. We generate a keyword-style search over the text corpus based on the cue words in the query and the most important context words of the Qfact (i.e., column headers and page title), plus the entity and quantity of the Qfact. Ideally, we would find witnesses $W$ with all these constituents, but we expect and handle also partial matches (as is the nature of keyword search) and approximate matches for the quantity value (e.g., rounded values or values within an interval, but ideally with the same unit). These considerations are cast into an evidence-score $(A, W)$ :

$\left(w_{1} \cdot \operatorname{sim}_{1}(A . c x t, W)+w_{2} \cdot \operatorname{sim}_{2}(\right.$ A.qty, W.qty) $) \cdot \operatorname{sim}_{3}($ A.ent, W.ent $)$

As some entities of the table-based Qfact repository may not appear in the text corpus at all, or merely in sophisticated text where they are hard to spot, we extend the search to other entities that match the query structure: same type, similar context cues, same quantity measure (disregarding the value). These same-type matches are viewed as confirming the proper query interpretation. Perfect matches of the Qfact entity itself carry higher weight than finding same-column entities; this is reflected by the entity-similarity weights in the above scoring function.

Top evidence scores per witness are averaged into an overall score for each candidate answer. The QuTE demo allows exploring the witnesses at query time. It involves additional text search and computation, but usually runs in a few seconds (after clicking on the "Evidence" button in the query result page).

Mutual Consistency of Answers. For a given query, we expect different answers to be consistent in two ways:

1. Their context cues are similar. For example, "worth", "value", "investment", etc. are consistent cues, but "salary", "taxes" etc. would be outliers for a query about the value of football teams.

2. Their quantity values should have limited variance. For example, team values of football clubs should be in the millions or billions, but not in the thousands or trillions.

To compute a consistency score, we adapt a randomized crossvalidation technique, originally proposed for image classification [17], which is completely self-supervised. Details are in [7]. The QuTE demo supports different configurations for this component.

Putting Everything Together. The three signals - matching score, evidence witnesses and mutual consistency - are combined by a weighted sum, with weights tuned via a small development set of gold-standard query-answer pairs.

\section{DEMONSTRATION}

QuTE is accessible at https://qsearch.mpi-inf.mpg.de/table/. Figure 2 shows a screenshot of top-ranked results for the example query "sprinters who ran 100 meters under 9.9s".

Data Sources. QuTE runs on a repository of ca. 18M Qfacts for ca. $800 \mathrm{~K}$ entities, extracted from two large corpora: WikiTables consisting of 1.8M tables from English Wikipedia, and TableL [8] consisting of $2.6 \mathrm{M}$ tables from $1.5 \mathrm{M}$ Common Crawl web pages.

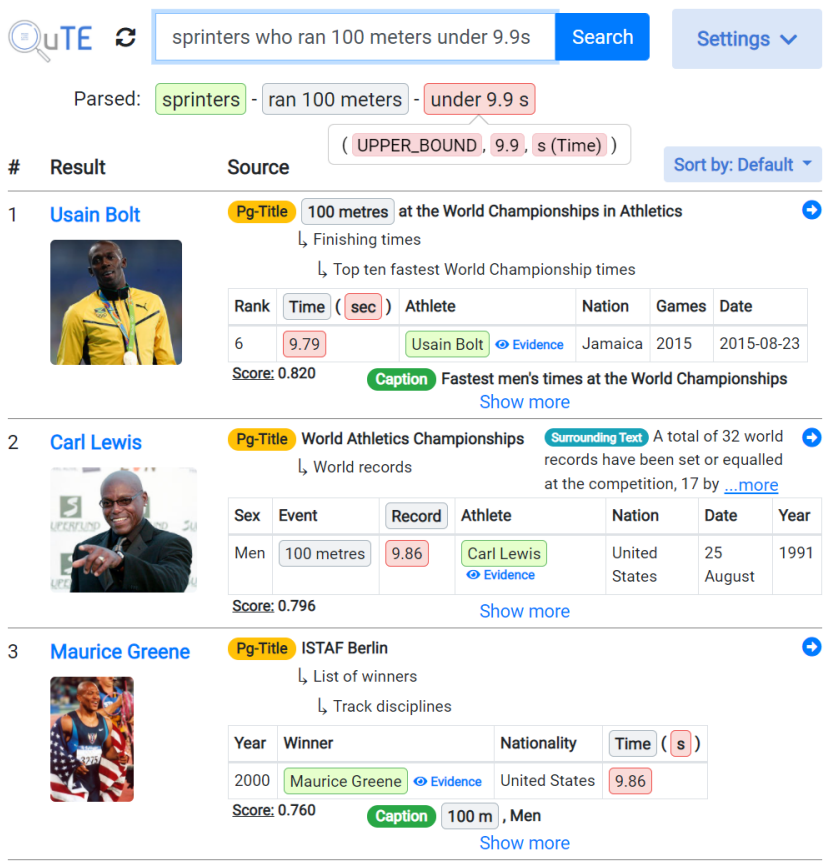

Figure 2: QuTE query and top-ranked answers.

The text-based witnesses draw from a text corpus of ca. $13.5 \mathrm{M}$ documents from Wikipedia and news articles.

Input. QuTE accepts user input in two modes: form-based and freetext. In form-based mode, users enter a query into three text fields (entity-type, quantity-filter, context-cues), with auto-completion suggestions for types. This mode helps our engine to evaluate the query as precisely as possible. In free-text mode, user queries are telegraphic phrases or full-fledged questions, which are then decomposed into the three constituents by a rule-based parser, as shown in the demo. The parser uses the Yago type taxonomy and a dictionary of quantity units.

To switch between the two input modes, users click on the toggle button on the left side of the search bar.

Output. QuTE computes a ranked list of entity answers, based on their confidence scores (see Section 4). The parsed query and top-k answers are shown to the users, along with context cues for each answer. Different colors are used to highlight answer entity (in light green), answer quantity (in red), column headers (obvious), page title (in orange), DOM-tree headings (in orange), table caption (in green), table row (obvious) and a surrounding-text snippet (in cyan), when available. By default, QuTE shows only the highest-scoring Qfact for each distinct entity. By clicking on the "Show more" button, additional Qfacts for the same entity can be displayed. These often include a noisy tail, showing the difficulty of the task.

Table 2 shows more anecdotal examples of quantity queries and their top answers produced by QuTE. For comparison, the table also shows top-3 results from a major search engine (SE), with incorrect, uninformative or very partial results in purple. These are evidence that search engines do not properly interpret quantity queries and simply return string-level matches. Some are high-quality list pages: browsing through them would give the user good results, but this 
Table 2: Queries with top-3 answers by QuTE vs. search engine (as of March 5, 2021). Purple answers are uninformative or very partial.

\begin{tabular}{l|l}
\hline Query: & Buildings higher than 500 meters \\
\hline QuTE & Burj Khalifa, One World Trade Center, Taipei 101, etc. \\
\hline SE & $\begin{array}{l}\text { List of tallest structures - 400 to 500 metres - Wikipedia, } \\
\text { List of tallest structures - Wikipedia, } \\
\text { China bans skyscrapers taller than 500 meters - Global Times }\end{array}$ \\
\hline \hline Query: & Which universities have more than 60000 students? \\
\hline QuTE & $\begin{array}{l}\text { Liberty University, University of Belgrade, } \\
\text { University of Toronto, etc. }\end{array}$ \\
\hline SE & $\begin{array}{l}\text { List of United States public university campuses by enrollment, } \\
\text { The top 50 US colleges that pay off the most 2019, } \\
\text { There Are Now 50 Colleges That Charge More Than } \$ 60000\end{array}$ \\
\hline \hline Query: Stadiums with more than 100000 seats \\
\hline QuTE & $\begin{array}{l}\text { Melbourne Cricket Ground, Rungrado 1st of May Stadium, } \\
\text { Tiger Stadium (LSU), etc. }\end{array}$ \\
\hline SE & $\begin{array}{l}\text { List of stadiums by capacity - Wikipedia, } \\
\text { List of European stadiums by capacity - Wikipedia, } \\
\text { World's Largest Sport Stadiums - Topend Sports }\end{array}$ \\
\hline
\end{tabular}

requires extra effort. Moreover, even when top-ranked results are useful, there is often a bias towards specific selections (e.g., US colleges although the query intent is world-wide).

The full paper [7] reports comprehensive experiments, showing that QuTE substantially outperforms standard search engines and other baselines in both precision and recall. This is even when the search engine is given the advantage of expanding list pages into entity answers, assuming that the user accepts the burden of manual browsing. Query run-times are usually within a few seconds; this could be accelerated by more code tuning.

Exploring Contextual Cues. Matching query cues in Qfact contexts is a vital element and novelty of QuTE. Consider the example shown in Figure 2. If all matching would be based on table rows alone, we could merely return Carl Lewis as a query result. If the matching were further restricted to the entity-quantity pairs alone (as it was pursued in prior works on knowledge extraction from web tables), even that result would be missing. The other two results, on ranks 1 and 3, would be completely out of scope. It is only by cues from page title, table caption, etc. that QuTE is able to discover these answers and rank them highly. Note that this entails identifying the beneficial cues in the Qfact context, among many uninformative or query-irrelevant ones.

Exploring Text-based Evidence. The witnesses from the large text corpus contribute to the overall scoring of QuTE answers (see Section 4). In addition, they are often interesting for users to obtain explanations and additional information. To showcase both the internal workings of QuTE and to support users in answer exploration, the demo allows viewing the witnesses by clicking on the "Evidence" button next to each answer entity. Figure 3 shows this for the answer Usain Bolt for the example query of Figure 2. The screenshot shows evidence snippets for the answer entity that match the query structure. We highlight the entities, the quantities, and the matching context words in the text evidence, e.g., Usain Bolt, 9.79 seconds, \{time, run, ran, 100 metres, ... $\}$.

Exploration of Different Configurations. Users can customize the search through a settings panel. QuTE can answer queries using either WikiTables or TableL or both corpora together (default).

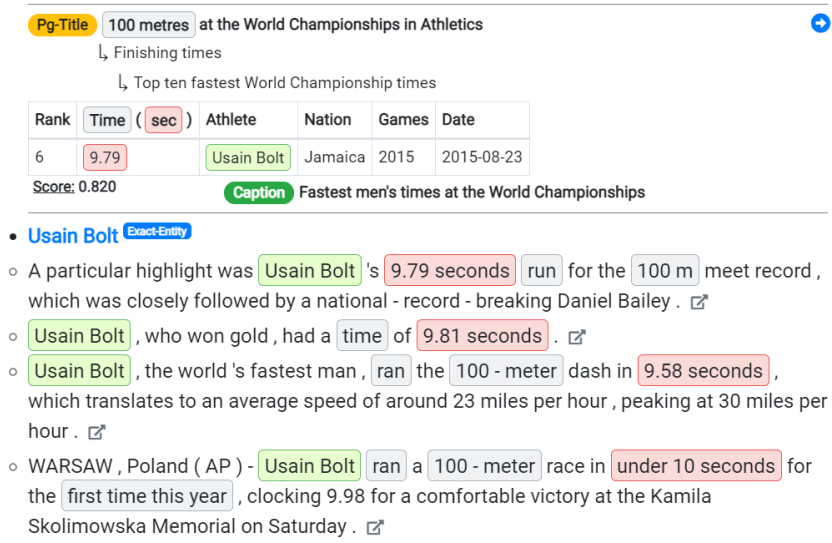

Figure 3: Evidence for the Qfact answer about Usain Bolt.

Further options are to customize the settings for column alignment and for consistency-based corroboration (see Section 4 and [7]).

By default, QuTE answers are ranked by confidence score. Users can re-rank them in ascending or descending order of quantity value, or by entity prominence via clicking on the "Sort by" button.

Limitations and Outlook. QuTE brings database-like queries to web contents: functionality that would be easy to express in SQL if the data resided in a single DB, but is novel for ad-hoc web tables. Being a prototype system, QuTE inevitably has notable limitations. The query parser for decomposing user input in free-text mode uses rules that may fail on certain phrases or miss out on certain kinds of quantities. The form-based input mode could possibly alleviate these issues, but is far from perfect either. We plan to enhance QuTE by expanding its library of measures and units, and by adding awareness of temporal scoping.

Another limitation is that QuTE can handle only queries with one quantity filter. For example, "stadiums with more than 100k seats built after 2000 " is beyond the current scope. We are working on extensions, to address such queries as well as support for group-by (e.g., " $100 \mathrm{~m}$ sprinters with more than 5 races under 9.9s").

\section{RELATED WORK}

Entity-centric knowledge extraction from web tables has been intensively explored (see, e.g., [2, 3, 9, 11, 18]). However, a common assumption has been that each table has a single subject column to which all other columns refer. This does not hold for complex tables such as the example in Table 1. Moreover, to support search over quantity properties, the bare extraction is insufficient, as it lacks contextualization with cue words that relate to user queries.

Methods for detecting numerical expressions (with units) have been developed $[1,12,13]$. However, inferring to which entity a detected quantity refers in a complex table row or sophisticated text passage, is out of scope for these methods. Our recent works $[5,7]$ close this gap.

Question answering over tables has been addressed from different angles, like translation into structured queries, machine learning for answer prediction, linkage with knowledge bases, and more (e.g., [4, 10, 13-16]). Closest to our work is [13], which has limited support for quantity filters, though. 


\section{REFERENCES}

[1] O. Alonso and T. Sellam. Quantitative information extraction from social data In The 41st International ACM SIGIR Conference on Research \& Development in Information Retrieval, SIGIR 2018, Ann Arbor, MI, USA, fuly 08-12, 2018, pages 1005-1008, 2018.

[2] C. S. Bhagavatula, T. Noraset, and D. Downey. Tabel: Entity linking in web tables. In The Semantic Web - ISWC 2015 - 14th International Semantic Web Conference, Bethlehem, PA, USA, October 11-15, 2015, Proceedings, Part I, pages 425-441, 2015.

[3] M. J. Cafarella, A. Y. Halevy, H. Lee, J. Madhavan, C. Yu, D. Z. Wang, and E. Wu. Ten years of webtables. Proc. VLDB Endow., 11(12):2140-2149, 2018.

[4] K. Chakrabarti, Z. Chen, S. Shakeri, G. Cao, and S. Chaudhuri. Tableqna: Answering list intent queries with web tables. CoRR, abs/2001.04828, 2020.

[5] V. T. Ho, Y. Ibrahim, K. Pal, K. Berberich, and G. Weikum. Osearch: Answering quantity queries from text. In The Semantic Web - ISWC 2019 - 18th International Semantic Web Conference, Auckland, New Zealand, October 26-30, 2019, Proceedings, Part I, volume 11778 of Lecture Notes in Computer Science, pages 237-257. Springer 2019.

[6] V. T. Ho, K. Pal, N. Kleer, K. Berberich, and G. Weikum. Entities with quantities: Extraction, search, and ranking. In WSDM '20: The Thirteenth ACM International Conference on Web Search and Data Mining, Houston, TX, USA, February 3-7, 2020, pages 833-836, 2020.

[7] V. T. Ho, K. Pal, S. Razniewski, K. Berberich, and G. Weikum. Extracting contextualized quantity facts from web tables. In $W W W^{\prime} 21$ : The Web Conference 2021, Ljubljana, Slovenia, April 19-23, 2021.

[8] Y. Ibrahim, M. Riedewald, G. Weikum, and D. Zeinalipour-Yazti. Bridging quantities in tables and text. In 35th IEEE International Conference on Data Engineering, ICDE 2019, Macao, China, April 8-11, 2019, pages 1010-1021. IEEE, 2019.
[9] G. Limaye, S. Sarawagi, and S. Chakrabarti. Annotating and searching web tables using entities, types and relationships. Proc. VLDB Endow., 3(1):1338-1347, 2010.

[10] B. Oguz, X. Chen, V. Karpukhin, S. Peshterliev, D. Okhonko, M. S. Schlichtkrull, S. Gupta, Y. Mehdad, and S. Yih. Unified open-domain question answering with structured and unstructured knowledge. CoRR, abs/2012.14610, 2020.

[11] Y. Oulabi and C. Bizer. Extending cross-domain knowledge bases with long tail entities using web table data. In Advances in Database Technology - 22nd International Conference on Extending Database Technology, EDBT 2019, Lisbon, Portugal, March 26-29, 2019, pages 385-396, 2019.

[12] S. Roy, T. Vieira, and D. Roth. Reasoning about quantities in natural language. Transactions of the Association for Computational Linguistics, 3:1-13, 2015.

[13] S. Sarawagi and S. Chakrabarti. Open-domain quantity queries on web tables: annotation, response, and consensus models. In The 20th ACM SIGKDD International Conference on Knowledge Discovery and Data Mining, KDD '14, New York, NY, USA - August 24 - 27, 2014, pages 711-720, 2014.

[14] J. Sen, C. Lei, A. Quamar, F. Özcan, V. Efthymiou, A. Dalmia, G. Stager, A. R. Mittal, D. Saha, and K. Sankaranarayanan. ATHENA++: natural language querying for complex nested SQL queries. Proc. VLDB Endow., 13(11):2747-2759, 2020.

[15] H. Sun, H. Ma, X. He, W. Yih, Y. Su, and X. Yan. Table cell search for question answering. In Proceedings of the 25th International Conference on World Wide Web, WWW 2016, Montreal, Canada, April 11 - 15, 2016, pages 771-782, 2016.

[16] J. Thorne, M. Yazdani, M. Saeidi, F. Silvestri, S. Riedel, and A. Y. Halevy. Neural databases. CoRR, abs/2010.06973, 2020.

[17] J. Yagnik and A. Islam. Learning people annotation from the web via consistency learning. In Proceedings of the 9th ACM SIGMM International Workshop on Multimedia Information Retrieval, MIR 2007, Augsburg, Bavaria, Germany, September 24-29, 2007, pages 285-290, 2007.

[18] S. Zhang and K. Balog. Web table extraction, retrieval, and augmentation: A survey. ACM Trans. Intell. Syst. Technol., 11(2):13:1-13:35, 2020. 